\title{
Rigidity percolation in a field *
}

\author{
Cristian F. Moukarzel ${ }^{\dagger}$ \\ Depto. de Física Aplicada, CINVESTAV del IPN, \\ Av. Tecnológico Km 6, 97310 Mérida, Yucatán, México
}

(Dated: October 30, 2018)

\begin{abstract}
Rigidity Percolation with $g$ degrees of freedom per site is analyzed on randomly diluted ErdősRenyi graphs with average connectivity $\gamma$, in the presence of a field $h$. In the $(\gamma, h)$ plane, the rigid and flexible phases are separated by a line of first-order transitions whose location is determined exactly. This line ends at a critical point with classical critical exponents. Analytic expressions are given for the densities $n_{F}$ of uncanceled degrees of freedom and $\gamma_{r}$ of redundant bonds. Upon crossing the coexistence line, $\gamma_{r}$ and $n_{F}$ are continuous, although their first derivatives are discontinuous. We extend, for the case of nonzero field, a recently proposed hypothesis, namely that the density of uncanceled degrees of freedom is a "free energy" for Rigidity Percolation. Analytic expressions are obtained for the energy, entropy, and specific heat. Some analogies with a liquid-vapor transition are discussed.

Particularizing to zero field, we find that the existence of a $(g+1)$-core is a necessary condition for rigidity percolation with $g$ degrees of freedom. At the transition point $\gamma_{c}$, Maxwell counting of degrees of freedom is exact on the rigid cluster and on the $(g+1)$-rigid-core, i.e. the average coordination of these subgraphs is exactly $2 g$, although $\gamma_{c}$, the average coordination of the whole system, is smaller than $2 g . \gamma_{c}$ is found to converge to $2 g$ for large $g$, i.e. in this limit Maxwell counting is exact globally as well.
\end{abstract}

PACS numbers: 05.70.Fh, 64.60.Ak, 02.10.Ox

\section{INTRODUCTION}

Scalar Percolation(SP) 1, 2] is a paradigm for the geometric phase transition that takes place on an initially disconnected lattice of point-like sites, when the density $p$ of present bonds is continuously increased. At the percolation point $p_{c}$ the system becomes connected on a macroscopic scale. This may mean that transport can happen across the system (conductivity, fluid flow), or that the system becomes correlated on macroscopic scales, i.e. ordered. It is in fact possible to describe magnetic transitions in terms of the percolation of properly defined clusters $[3,4,5]$. Because of the generality and simplicity of the concepts involved, this paradigm has found multiple applications in science [1, 2, 6, 7]. In most cases, the physical variables attached to sites are scalars, i.e. each site has one associated degree of freedom.

A generalization of this paradigm considers the case in which there is more than one degree of freedom per site, and has been termed Rigidity Percolation(RP) 8, 9, 10, 11, 12, 13, 14, 15]. In $g$-RP, each site of a lattice has $g$ degrees of freedom, and each present bond eliminates one relative degree of freedom. The most commonly invoked application of RP deals with the statics of structures. Consider for example the problem of bracing a framework in three dimensions, i.e. rigidly connecting a set of point-like joints by means of rotatable bars. Each joint has three translational degrees of freedom, $g=3$, and

*Dedicated to Dietrich Stauffer, on the occasion of his 60th birthday.

†email address: cristian@mda.cinvestav.mx each rotatable bar fixes the distance between two nodes, thus providing one relative constraint. The question of whether a given set of bars is enough to rigidize a given structure constitutes a classical problem in applied mathematics, that of Graph Rigidity [16, 17, 18]. In statistical physics, bonds (bars) are randomly present with probability $p$ or absent with probability $1-p$, and one asks for the typical rigid properties of such structures. Upon increasing the density $p$ of present bonds, the system goes into the rigid phase, characterized by the existence of an extensive rigidly connected cluster. The RP problem becomes fully equivalent to SP when $g=1$. SP has a continuous transition in all dimensions 1]. In two dimensions the RP transition is continuous but in a different universality class 60 than SP. In the Mean Field(MF) limit, $\operatorname{RP}(g>1)$ has a first-order transition [19, 20].

The situation is reminiscent of the Potts model, whose MF transition is continuous for $q=2$ and discontinuous for $q>2$ 21, 22]. Potts models in the presence of a field, and their relation with percolation models, have been studied recently because of possible links with the deconfining transition in QCD [23, 24]. In the presence of a field, and for large enough $q$, the Potts model has a line of first-order transitions ending at a critical point [25, 26]. This critical point appears to always be in the Ising universality class.

Nonzero field values have been considered in scalar percolation studies previously [27, 28, 29, 30, 31, 32, 33]. A field may be introduced in percolation by allowing for the existence of "ghost bonds" which are present with probability $h$ and connect sites directly to a solid background (or to "infinity"). However, for any nonzero field there is no SP transition. RP is somewhat more interesting, as we will find out. In this work, RP is studied on 
diluted random graphs of the Erdős-Renyi type, with average connectivity $\gamma$, in the presence of a field $h$. Unlike $\mathrm{SP}$, mean-field RP has, in the presence of a field, a line of first-order phase transitions. This line ends at a critical point with classical critical indices: $\alpha=0, \beta=1 / 2$, $\gamma=1$ and $\delta=3$.

Some of the analysis in zero field is relevant for the related problem of Bootstrap Percolation (BP) 34, 35, 36, 37], also known as $k$-core 38 in the field of Graph Theory [39, 40, 41]. In $k$-BP [34], all sites with less than $k$ neighbors are iteratively culled. What remains, if something, is the $k$-core [38]; a subgraph were all sites have $k$ or more neighbors. In $g$-RP a site needs at least $g$ bonds in order to be attached to a rigid cluster. Thus the "infinite" rigid cluster is a subset of the $g$-core. In Section IB we will see that an even stronger condition exists for rigidity: $g$-RP requires the existence of a $(g+1)$-core [61].

Our approach starts by deriving an equation of state for the "order parameter" $R(\gamma, h)$, the probability that a randomly chosen site belongs to the rigid cluster, as a function of $\gamma$, the average number of bonds impinging on a site, and $h=\gamma H$, where $H$ is the applied field. We will call $H$ or $h$ indistinctly the "field" variable. The equation of state, as is customary in these cases, is found to accept multiple solutions. Stability analysis is not enough to single out a unique solution. Of central importance in order to lift this multiplicity are $\gamma_{r}(\gamma, h)$, the average number of "redundant" bonds per site (see later), and $n_{F}$, the average number of uncanceled degrees of freedom per site. Their relevance resides in the fact that they must be continuous functions of $\gamma$. Requiring that $n_{F}$ (or, equivalently, $\gamma_{r}$ ) be continuous is enough to identify the physically correct solution.

This work is similar in spirit to previous treatments of $\mathrm{RP}$ in zero field on Bethe lattices [20], i.e networks where each site has exactly $z$ randomly chosen neighbors. We consider the effect of an external field, and particularize to random graphs of the Erdös-Renyi type 42. The introduction of a field appears to be much more tractable analytically on Erdős-Renyi graphs than on Bethe lattices, and this is the main reason why most of the results presented here are for Erdős-Renyi graphs. For these, we are able to derive analytic expression for the densities $n_{F}$ of uncanceled degrees of freedom and $\gamma_{r}$ of redundant bonds for arbitrary values of the field. However in zero field calculations are also straightforward for Bethe lattices, and allow us to obtain, in an entirely analytic fashion, some of the results obtained by numerical integration in Ref. 20].

It is known that SP and Potts models are particular cases of a more general Fortuin-Kasteleyn RandomCluster(RC) model, defined by a continuous parameter $q$ [43, 44]. SP can be obtained as the $q \rightarrow 1$ limit of this Random Cluster model and, in this limit, the logarithm of the partition function coincides with the average number of connected clusters. It seems possible that a similar mapping might exist for RP as well, although it has not been found up to now. However it has been proposed [20, 45] that the number of uncanceled degrees of freedom $n_{F}$ is a good "free energy" candidate for RP in zero field. When $g=1$, each connected cluster has one uncanceled degree of freedom, so both definitions coincide in this limit. We explore on this idea further in this work.

It is possible to establish a pedagogical parallel between the RP transition and a Condensation transition. One identifies the coordination parameter $\gamma$ with an inverse temperature $\beta$; the negative of the order parameter $R$ plays the role of the fluid volume $V$ (it is also possible to identify $R$ with $\rho$, the fluid density) 62], and the field $H=h / \gamma$ is the fluid pressure $P$. Within this analogy, it results natural to argue that requiring the continuity of $n_{F}$ in order to identify the physically correct solution $R$ in Rigidity Percolation is equivalent to requiring the continuity of the free energy (giving rise to the Maxwell construction) in the Statistical Mechanics treatment of condensation transitions at the MF level [46].

It is shown in this work that the idea of identifying $n_{F}$ with a free energy for RP leads to consistent results in the presence of a field as well. In Section $\nabla 1$ it is shown that: 1) the order parameter $R$ is obtained as a derivative of the free energy with respect to the field, 2) the condition of stability (Section IIIC) may be related to the positivity of a suitable second derivative of the free energy, and, 3) the continuity of $n_{F}$ can be cast exactly in the form of Maxwell's Rule of Equal Areas on the RP equivalent of a $P-V$ diagram (which is the $H-R$ diagram, see Fig. (7).

This work is organized as follows: The system under consideration is defined in Section [IA and its equation of state is derived in Section $\amalg B$ establishing its connections with Bootstrap Percolation. In Section【C the field is introduced. Section [II] starts the analysis of solutions of the general equation of state, discussing stability and the existence of a critical point. The concept of redundant constraints is introduced in Section IV A and their density is calculated in Section IVB This result is used in Section $\nabla$ to determine the value $\gamma_{c}$ where the firstorder transition takes place. In Section $\nabla B$ the counting of constraints is done on the $(g+1)$-rigid core in zero field, both for Erdős-Renyi graphs and for Bethe lattices. Section [V] discusses several consequences of identifying the density of uncanceled degrees of freedom with a free energy, and Section VII contains a discussion of the results.

\section{SETUP}

\section{A. Randomly diluted graphs}

We consider graphs made of $N$ sites (or "nodes") where each of the $N(N-1) / 2$ pairs of distinct sites is connected by a bond (or "edge") independently with probability $p$. This defines [42] an Erdös-Renyi graph with average coordination number $\gamma=p(N-1)$. In this work $\gamma$ will be 
taken to be of order one. For large $N$, a site of this graph is connected to $k$ other sites with poissonian probability $P_{k}(\gamma)=e^{-\gamma} \gamma^{k} / k !$.

As appropriate for Rigidity Percolation, each node of this graph is regarded as a "body" with $g$ degrees of freedom. For example, rigid bodies in $d$ dimensions have $d$ translational degrees of freedom plus $d(d-1) / 2$ rotational degrees of freedom, for a total of $g=d(d+1) / 2$. In this work, $g$ is taken to be an arbitrary integer. Each present bond represents one constraint, that is, removes one degree of freedom. In order to have a physical representation in mind, a bond can be though of as a rotatable bar that fixes the distance between two arbitrary points belonging to the bodies it connects 15, 47. For the class of graphs that we consider, at most one bond is allowed for each pair of nodes.

As the number of present bonds grows, parts of this graph will become "rigidly connected". Rigid connectivity of a subgraph means (this is our definition) that the total number of degrees of freedom in the system cannot be further reduced by additional bonds connected between nodes of this subgraph. Such subgraphs are customarily called "rigid clusters". A rigid cluster has no internal degrees of freedom left. When the coordination $\gamma$ is large enough, the largest rigid cluster encloses a finite fraction of the system's sites, and rigidity is said to percolate. For low $\gamma$ the system is in the flexible, or floppy, phase, and there are no extensive rigid clusters.

A rough estimate of the threshold for the appearance of an extensive rigid cluster can be obtained by equating the average number of constraints per site, which is $\gamma / 2$, to $g$. This results in the so called Maxwell estimate 13, 20, 45, 48], $\gamma_{c}^{\text {Maxwell }}=2 g$. This estimate becomes exact in the $g>>1$ limit (Section $\nabla \mathrm{B}$ ).

\section{B. The Equation of State in zero field}

Let us now define $\mathcal{C}$ to be the largest rigid cluster in the graph, if one exists. A site in $\mathcal{C}$ will be said to be a "rigid site". Let $R$ be the probability that a randomly chosen node $i$ be in $\mathcal{C}$. In order for $i$ to be in $\mathcal{C}$ it is necessary and sufficient that it be connected to $g$ or more other nodes $j$ in $\mathcal{C}$. Our definition of rigidity is thus recursive at this stage.

A site $j$ is rigid and connected to $i$ with probability $p R$. Therefore $i$ has exactly $k$ rigid neighbors with probability $\left(\begin{array}{c}N-1 \\ k\end{array}\right)(p R)^{k}(1-p R)^{N-1-k}$. For $N$ large and defining $x=\gamma R$, the probability $P_{k}$ to have exactly $k$ rigid neighbors may be written as

$$
P_{k}(x)=e^{-x} x^{k} / k !
$$

showing that the number $k$ of rigid neighbors of a randomly chosen site is a poissonian variable with average $x=\gamma R=\sum_{k=0}^{\infty} k P_{k}(x)$. Since a site must have $g$ or more rigid neighbors in order to be itself rigid, we conclude that
$R$ satisfies the self-consistent equation:

$$
R=\sum_{k=g}^{\infty} P_{k}(\gamma R)=G_{g}(\gamma R)
$$

where we have defined

$$
G_{m}(x)=\sum_{k=m}^{\infty} P_{k}(x) .
$$

\section{Bootstrap Percolation, or $k$-core}

Let us now briefly discuss the related problem of Bootstrap Percolation [34] or $k$-core [38]. We want to assess the probability $B P(g+1)$ that a randomly chosen site $i$ be part of the $(g+1)$-core. Assume this is the case. By following one of its links, a neighbor $j$ is reached. $j$ must have at least $g$ other neighbors. We call these neighbors of $j$, other than the site $i$ from which we arrived at it, the "outgoing" neighbors of $j$. Each of these in turn must have $g$ or more outgoing neighbors, and so on. More formally, let us define the property of $g$-outgoingconnectedness $(g$-OC) in the following (recursive) way: A site is $g$-OC if $g$ or more of its outgoing neighbors also are.

On graphs of the Erdős-Renyi type, the probability to have $k$ outgoing neighbors is the same as that to have $k$ neighbors altogether, i.e. $P_{k}(\gamma)$ (Eq. (1)). This is so because links are independently present. Letting $\hat{R}$ be the probability that a site be $g$-OC, by the same reasoning as in the previous Section we conclude that a random site is connected to exactly $k$ outgoing $g$-OC neighbors with probability $P_{k}(\gamma \hat{R})$. Thus $\hat{R}$ satisfies the same selfconsistent equation (2) as $R$ does in the RP problem with $g$ degrees of freedom, i.e.

$$
\hat{R}=G_{g}(\gamma \hat{R})
$$

Notice that, by requiring that each site in a tree have $g$ or more $g$-OC neighbors we ensure that all sites, except perhaps for the top one, have $(g+1)$ or more neighbors. The probability $B P(g+1)$ that the top site itself has $g+1$ or more neighbors (which are $g$-OC) is then given by

$$
B P(g+1)=G_{g+1}(\gamma \hat{R})=\hat{R}-P_{g}(\gamma \hat{R}) .
$$

This expression gives the density of the $(g+1)$-BP infinite cluster, (or $(g+1)$-core) at the point where it first appears 34, 39, 41]. So one must first solve (4) in order to obtain $\hat{R}$ as a function of $\gamma$, and then use (5) to find $B P(g+1)$. Numerical results [63] show that (5]) is exact for large $N$.

We see that the $(g+1)$-core density $P B(g+1)$ is somewhat smaller than $\hat{R}$ whenever $\hat{R}>0$, while $\hat{R}$ in turn satisfies the same equation as the density $R$ of rigid sites in $g$-RP. Later in Section $\mathrm{VB}$ we will see that in zero field, whenever there is a $g$-rigid cluster, it contains as a subset the $(g+1)$-core. 


\section{Equation of State in the presence of a ghost field}

We now introduce a "ghost field" $H$ that couples to the order parameter $R$, in the following way [32]: in addition to the "normal" bonds of our graph, a number $N \gamma H=N h$ of "ghost-bonds" are assumed to exist, each connecting one site to a unique rigid background [64]. Each ghost bond provides one constraint, i.e. removes one degree of freedom. Multiple occupation is allowed, so that a random site is connected to the background by $n$ ghost bonds with poissonian probability $P_{n}(h)=e^{-h} h^{n} / n !$.

It is easy to see that all extensive rigid clusters are rigidly connected to the background. From now on, a site is said to be rigid if it is rigidly connected to the background, either directly through ghost bonds, or indirectly through rigid neighbors.

If a site has $n \geq g$ ghost edges connecting it to the background, then it is rigid. Otherwise if $n<g$, it is rigid if it has, in addition to these $n$ ghost bonds, $(g-n)$ or more rigid neighbors. Thus using (11) we may write, in the presence of a field $h=H \gamma$,

$$
R=\sum_{n=g}^{\infty} P_{n}(h)+\sum_{n=0}^{g-1} P_{n}(h) \sum_{k=g-n}^{\infty} P_{k}(\gamma R)
$$

After a simple resummation, this expression reads

$$
R=G_{g}(\gamma R+h),
$$

with $G_{g}$ given by (3). Eq. (7) generalizes (2) in the presence of a field, and is the equation of state for our problem.

The simplicity of (7) is one of the reasons leading us to study this particular (Poissonian) definition of the field. Other field definitions, like for example assuming that each site is rigidly attached to the background with probability $h$, or other random graph structures like Bethe lattices 20] are also tractable with the methods used here, but the algebra becomes more complicated.

Clearly $y=\gamma(H+R)$ plays the role of a "Weiss field" in the MF equation for a ferromagnet. By analogy we may thus identify $\gamma$ as the inverse temperature, and $H$ as the magnetic field. It is illustrative to take notice of the similarities between (7) and other MF equations. For the Potts model [4],

$$
m=\Theta_{q}(\beta m+h)
$$

where $\beta$ is the inverse temperature, $m$ is the magnetization, $h=\beta H$ is the external field, and $\Theta_{q}(y)=\left(e^{y}-1\right) /\left(e^{y}+(q-1)\right)$.

Notice that when $g=1$ (Scalar Percolation), (77) gives $G_{1}(y)=1-e^{-y}$, the same as (8) for $q=1$. This is of course just a consequence of the known equivalence between Scalar Percolation and the $q \rightarrow 1$ limit of the Potts model [43, 44].
However, the archetypal example of a first-order transition with a two-parameter phase space is the condensation transition 46], described at the Mean-Field level by the Van der Waals equation. The reduced form of the Van der Waals equation can be written as

$$
\lambda=\Gamma(\beta(\lambda+P))
$$

where $\Gamma(y)=27 y^{2} /(y+8)^{2}, P$ is the pressure, $\beta$ is the inverse temperature and $\lambda=3 \rho^{2}$ with $\rho$ the number density. $\Gamma$ has the same general features as $G$ and $\Theta$, namely it starts at zero, grows sharply, and saturates for large values of its argument. Therefore all three systems give rise to the same phenomenology, including of course sharing the same (classical) critical indices at their critical points.

Within an analogy with a condensation transition, in the $\mathrm{RP}$ problem $\gamma$ plays the role of an inverse temperature, while $H=h / \gamma$ plays the role of a pressure.

\section{ANALYSIS OF SOLUTIONS}

In this Section, the solutions of our equation of state (77) are discussed. In order to obtain $R(\gamma, h)$ numerically for given $\gamma$ and $h$, one might for example iterate (7) until a desired numerical accuracy is reached. This procedure was used in Refs [19, 20]. However in this work the following alternative procedure was preferred: given $h>0$ fixed, and for a sequence of values of $y>h$, evaluate

$$
\left\{\begin{array}{l}
R=G_{g}(y) \\
\gamma=(y-h) / G_{g}(y)
\end{array}\right.
$$

thus defining $R(\gamma, h)$ implicitly. In this way one obtains the results displayed in Fig. 11. This procedure allows us to obtain all solutions of (7), while the iterative procedure mentioned above only provides the stable branches.

In zero field, $R=0$ is a solution $\forall \gamma$ and $\forall g$. If $\gamma$ is large enough, nontrivial solutions $R>0$ exist as well. If $h>0$ one has on the other hand that $R>0 \forall \gamma$.

In the SP case $(g=1), G_{1}(x)=1-\exp (-x)$. Thus if $h=0$ a non-zero solution for $R$ first appears at $\gamma_{c}=1$. Above $\gamma_{c}$ one has $R \approx(\gamma-1)$. In this case there is a continuous transition at $\gamma=1$, a well known result for scalar percolation on random graphs [42]. If $h>0$ there is no transition (Fig. 1).

A richer behavior is found for the RP case $(g>1)$, as shown in Fig. 1 for $g=2$. In this case $\gamma$, as given by Eq. (10), may no longer be a monotonous function of $y$, and, for this reason, $R$ becomes a multivalued function of $\gamma$. This allows for the existence of a first-order transition. The physical considerations which lead to the identification of the correct transition point will be discussed in Sections IIIC and V. 

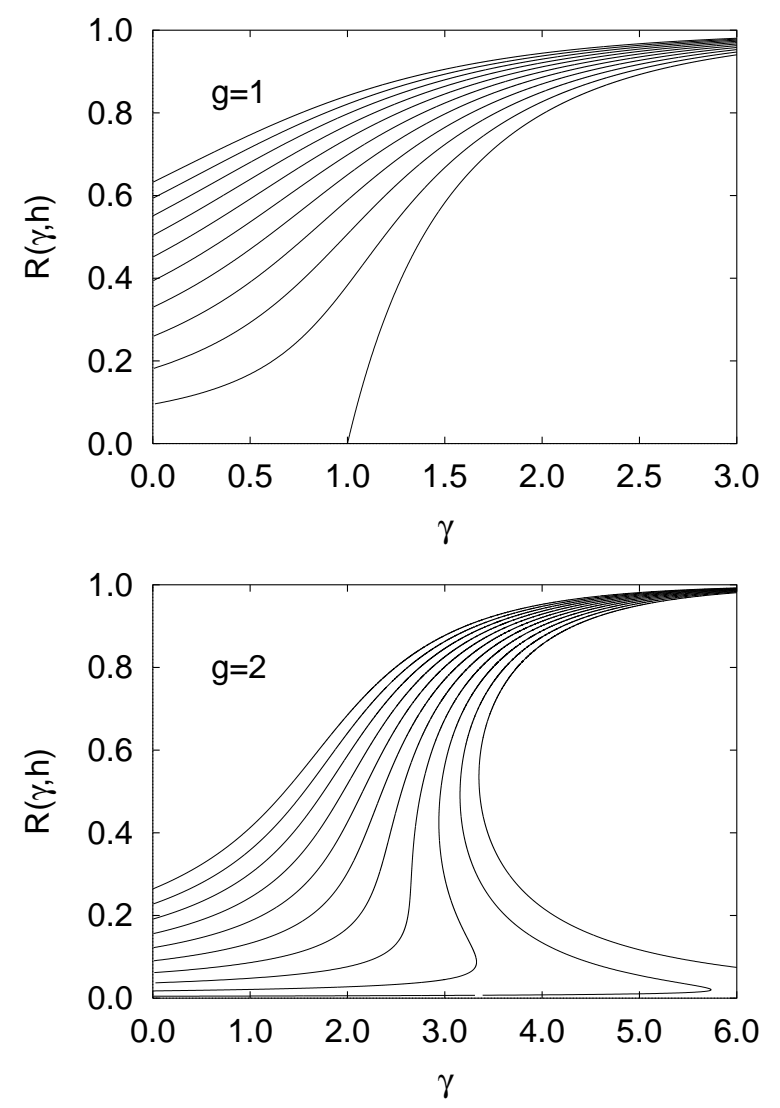

FIG. 1: The density $R$ of "rigid sites" as given by Eq. (7), for $g=1$ (scalar percolation, top) and $g=2$ (rigidity percolation, bottom). The field takes the values (from right to left): $h=$ $0.0,0.1,0.20, \ldots, 1.0$. For $g=2$ the critical field is $h_{c}=0.287$.

\section{A. Spinodal Points}

The condition that $\gamma$ as given by Eq. (10) be stationary in $x$ reads

$$
G_{g}-x \frac{\partial G_{g}}{\partial x}=0
$$

and has two solutions $x_{ \pm}^{s}(g, h)$ for all $h$ smaller than a critical value $h^{*}(g)$. These two solutions in turn define $\gamma_{ \pm}^{s}$, which are turning points for $R(\gamma, h)$ (See Fig. 2). In the interval $\gamma_{+}^{s}<\gamma<\gamma_{-}^{s}, R(\gamma, h)$ has three solutions, two of which are stable as we show next. Therefore a discontinuous $\gamma$-driven transition takes place when $h<$ $h^{*}(g)$. Right at $h=h^{*}$ the transition becomes continuous (See Section IIIB), while for $h>h^{*}(g), R$ is a smooth function of $\gamma$ and no transition occurs.

The thresholds $\gamma_{ \pm}^{s}$ can be identified as spinodal-points, as first discussed in [20]. The true rigidity percolation transition happens at a value $\gamma_{c}(g, h)$ that lies in between the spinodals, and which we analytically determine later in Section $\nabla$

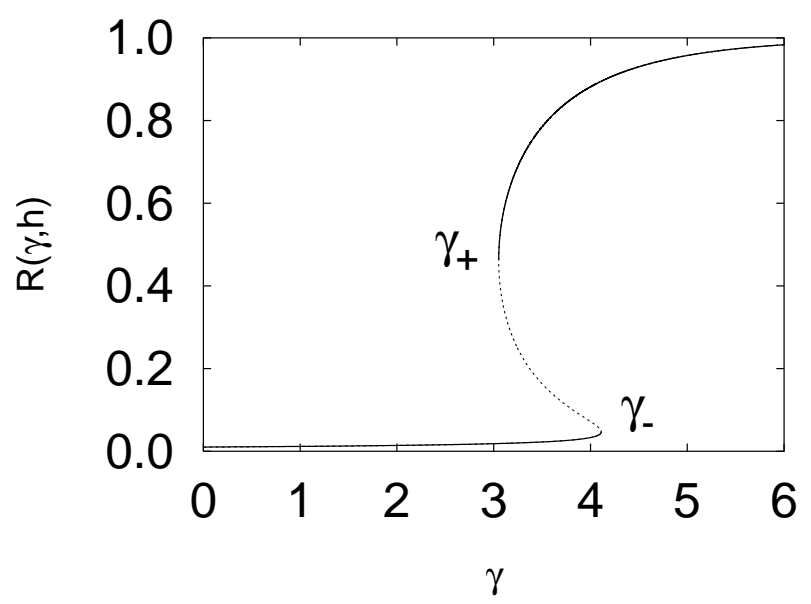

FIG. 2: For all $g>1$, the order parameter $R$ as given by (10) becomes multivalued for small values of the field $h$. The branch joining the spinodals $\gamma_{ \pm}$(dashed) has $d R / d \gamma<0$ and is thus unstable. The uppermost and lowermost branches (solid) are stable. In this example, $g=2$ and $h=0.15$. For zero field the spinodal $\gamma_{-}$goes to infinity, and the branch $0 \leq \gamma \leq \gamma_{-}$collapses onto the $R=0$ solution, which is stable for all $\gamma$.

\section{B. Critical Point}

When $h$ takes a critical value $h^{*}$, the spinodals $x_{ \pm}^{s}$ coalesce onto an inflexion point where $\partial G_{g}(x+h) / \partial x$ equals $G_{g} / x$ (since Eq. (11) holds). The critical point $\left\{h^{*}, \gamma^{*}, R^{*}\right\}$ is thus defined by

$$
\begin{aligned}
\left.\frac{\partial^{2} G_{g}}{\partial x^{2}}\right|_{x^{*}, h^{*}} & =0 \\
\left.\frac{\partial G_{g}}{\partial x}\right|_{x^{*}, h^{*}} & =\frac{G_{g}\left(x^{*}+h^{*}\right)}{x^{*}}=\frac{1}{\gamma^{*}} .
\end{aligned}
$$

For $h>h^{*}$ there is no phase transition. Using Eq. (7), and defining $y=x+h$, these two equations can be solved exactly. The critical point turns out to be

$$
\left\{\begin{array}{l}
y^{*}=g-1 \\
\gamma^{*}=\left\{P_{g-1}(g-1)\right\}^{-1}=e^{g-1}(g-1) ! /(g-1)^{g-1} \\
R^{*}=G_{g}(g-1)=e^{1-g} \sum_{k=g}^{\infty}(g-1)^{k} / k ! \\
h^{*}=y^{*}-x^{*}=g-1-\gamma^{*} R^{*}
\end{array}\right.
$$

For the scalar case $(g=1)$ this gives $h^{*}=R^{*}=0$ and $\gamma^{*}=1$ in accordance with the fact 42$]$ that for zero field there is a continuous transition at $\gamma_{c}=1$. 
For $g=2$ one finds

$$
\left\{\begin{array}{l}
\gamma^{*}=e \\
R^{*}=(e-2) / e \\
h^{*}=3-e .
\end{array}\right.
$$

For large $g$, and approximating $n ! \approx(n / e)^{n}(2 \pi n)^{1 / 2}$ one sees that $\gamma^{*} \propto g^{1 / 2}$, while $h^{*} \sim g$ and thus $H^{*} \sim g^{1 / 2}$. This means that in the limit $g>>1$ most constraints are field-constraints at the critical point.

\section{Critical Indices}

The RP transition on random graphs is similar to other MF transitions with first-order lines, as discussed in Section IC Thus it can be concluded that RP must have classical critical indices: $\beta=1 / 2, \delta=3$ and $\gamma=1$. For completeness we show that this is indeed the case by deriving the critical indices briefly in Appendix B

\section{Stability analysis}

Stability can be analyzed if Eq. (17) is interpreted as a recursion relation,

$$
R_{t+1}=G\left(\gamma R_{t}+h\right), \quad t=0,1, \ldots, \infty,
$$

for fixed $\gamma$ and $h$. Assume $R(\gamma, h)$ is a fixed point of Eq. (15), i.e. $R=G(\gamma R, h)$. This fixed point is stable if

$$
\left.\left|\frac{\partial G(\gamma R+h)}{\partial R}\right|_{\gamma, h}|=| \gamma \frac{\partial G(x+h)}{\partial x}\right|_{x=\gamma R} \mid<1 .
$$

Since $G^{\prime}(y)=P_{g-1}(y)$ we conclude that the stability condition reads

$$
\gamma P_{g-1}(y)<1,
$$

with $y=x+h$ as defined previously.

A more useful form results if one notices that, if $R>0$, Eq. (16) is equivalent to requiring that $d R / d \gamma>0$. In fact,

$$
\begin{gathered}
\frac{d R}{d \gamma}=\frac{d G}{d x} \frac{d x}{d \gamma}=G^{\prime} \frac{d}{d \gamma}(\gamma R)=G^{\prime}\left(R+\gamma \frac{d R}{d \gamma}\right) \\
\Rightarrow \quad \frac{d R}{d \gamma}\left(1-\gamma G^{\prime}\right)=G^{\prime} R
\end{gathered}
$$

and since $G^{\prime}>0 \forall x>0$, we conclude that a nontrivial fixed point $R(\gamma, h)>0$ of Eq. (15) is stable if and only if $d R / d \gamma>0$.

This condition has a simple physical meaning: increasing the average connectivity $\gamma$ should not decrease the rigid density $R$. A similar stability condition holds for fluids, namely that the coefficient of thermal expansion be positive.

\section{Scalar Percolation $(g=1)$}

In zero field, $R=0$ is a solution of Eq. (7), thus a fixed point of Eq. (15), $\forall g$. If $g=1$, stability (Eq. (17)) requires that $\gamma P_{0}=\gamma e^{-\gamma R}<1$. Thus the trivial solution $R=0$ becomes unstable for $\gamma>1$, where the nontrivial solution (stable because $d R / d \gamma>0$. See Fig. (1) first appears. This situation is typical of continuous transitions; the ordered solution appears exactly at the point where the paramagnetic solution becomes unstable.

When $h>0$ there is only one solution for Eq. (7), and it is stable for all $\gamma$ since $d R / d \gamma>0$ (Eq. (18)).

\section{Rigidity Percolation $(g>1)$}

For $g>1$ the situation is more interesting, as several stable solutions of Eq. (7) can coexist. If $h<h^{*}$, Eq. (7) has three solutions (see Fig. 2) in the range $\gamma_{-}^{s}>\gamma>\gamma_{+}^{s}$. The branch joining the two spinodal points can be discarded since it is unstable (because $d R / d \gamma<0$ ). However the other two branches (solid lines in Fig. 2) are stable so that there is coexistence of two stable solutions for $\gamma_{-}^{s}>\gamma>\gamma_{+}^{s}$. Thus the system undergoes a $\gamma$-driven first-order transition somewhere between the spinodals. The precise point $\gamma_{c}$ at which the system switches from one stable solution to the other is uniquely defined by a continuity requirement, as we elaborate later in Section $\mathbb{\nabla}$

The $h=0$ case is similar, however in this case the lowest stable branch collapses onto the trivial solution $R=0$, while the spinodal $\gamma_{-}$goes to infinity.

\section{ZERO MODES, REDUNDANT AND OVERCONSTRAINED BONDS}

In this Section the counting of uncanceled degrees of freedom and the useful notions of redundant and overconstrained bonds are discussed. These will be of central importance in our subsequent treatment of the RP problem.

\section{A. Definitions}

We consider a graph made of $N$ sites, each with $g$ degrees of freedom, and for the moment assume that $h=0$, i.e. there are no ghost-field constraints. Each present bond removes one degree of freedom from the system, unless it is a redundant bond. A redundant bond (or constraint) is one that links two nodes which were already rigidly connected, as for example nodes $i$ and $j$ in Fig. 3] The addition of a redundant constraint does not reduce the number of degrees of freedom in the system. Thus the balance of degrees of freedom reads

$$
N_{F}=N g-E+B_{r}
$$


where $N_{F}$ is the number of uncanceled degrees of freedom 65], $E$ is the number of bonds in the graph, and $B_{r}$ is the number of redundant bonds.

For an Erdős-Renyi graph one has $\langle E\rangle=\gamma N / 2$. Defining $\left\langle B_{r}\right\rangle=\gamma_{r} N / 2$, the average density $n_{F}(\gamma)$ of zero modes per site is written

$$
n_{F}(\gamma)=\frac{\left\langle N_{F}\right\rangle}{N}=g-\frac{1}{2}\left[\gamma-\gamma_{r}(\gamma)\right]
$$

Let us assume that $b$ is a redundant bond. This means

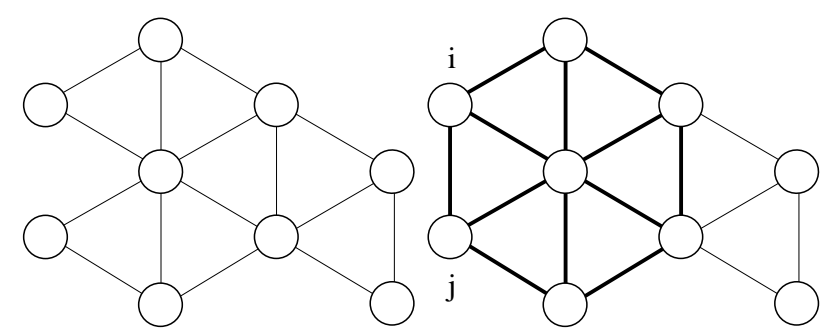

FIG. 3: In this two dimensional example, each node (circle) represents a point that has two positional degrees of freedom, while each bond fixes the distance between two points and thus provides one constraint. The graph on the left still has three remaining degrees of freedom: two translations and one rotation. Bond $i j$ (right) is a redundant bond, because it connects two nodes which were already rigidly connected. After adding bond $i j$, the bonds that provided the rigid connection between $i$ and $j$ become overconstrained (thick lines). Any one of these can be removed without altering the number of remaining degrees of freedom, which still equals three. The graph on the right has one redundancy, but 12 overconstrained bonds.

that its two end-nodes are rigidly connected even if $b$ is removed. Let $\mathcal{B}_{b}$ be the subset of bonds that, in the absence of $b$, provide rigidity to its two end-nodes. After adding $b$, any of the bonds in $\left\{\mathcal{B}_{b}+b\right\}$ (thick lines in Fig. 3) can be removed without altering the total number of degrees of freedom $N_{F}$. The subset of bonds $\left\{\mathcal{B}_{b}+b\right\}$ is said to be overconstrained. From a physical point of view, the overconstrained bonds may be defined as those that carry an internal stress because of the addition of a redundant bond $b$ that has a length-mismatch.

Please notice the important difference between the number of redundancies and the number of overconstrained bonds: when adding a redundant bond (like $i j$ in Fig. 3 ) the number of redundancies $B_{r}$ always increases by exactly one. However the number of overconstrained bonds may increase by more than one. In the example shown in Fig. 3] the number of overconstrained bonds increases by twelve.

We then conclude that, when adding any bond to a graph, the number of redundancies $B_{r}$ will either increase by one (if the chosen sites were rigidly connected) or stay unchanged (if not), in which case the number of zero modes $N_{F}$ will either stay unchanged or decrease by one. This implies that the densities $\gamma_{r}$ of redundant bonds and $n_{F}$ of zero modes must be continuous functions of the density $\gamma$ of present bonds. The density of overconstrained bonds, on the other hand, needs not be continuous.

Let us now discuss how the balance of degrees of freedom is modified by ghost-field bonds (Section IIC). If a site has $n<g$ present ghost edges connecting it to the rigid background, then it contributes with $(g-n)$ degrees of freedom to Eq. (19). If on the other hand it is connected to the background by $n \geq g$ ghost bonds, then this site has no degrees of freedom left to contribute to Eq. (19). The effective number $g_{\text {eff }}(h)$ of remaining degrees of freedom contributed by an average site is thus

$$
\begin{aligned}
g_{\mathrm{eff}}(h) & =\sum_{n=0}^{g}(g-n) P_{n}(h) \\
& =g\left(1-G_{g+1}(h)\right)-h\left(1-G_{g}(h)\right),
\end{aligned}
$$

where $G_{g}$ is defined by (3). Taking this result into account, in the presence of a field $h$ we now have that

$$
n_{F}(\gamma, h)=g_{\mathrm{eff}}(h)-\frac{1}{2}\left[\gamma-\gamma_{r}(\gamma, h)\right] .
$$

Notice that $n_{F}$ and $\gamma_{r}$ are trivially related through the addition of a known function of $\gamma$ and $h$. Therefore the knowledge of either $n_{F}$ or $\gamma_{r}$ provides exactly the same physical information about the system.

The importance of (22) is twofold: in the first place, as discussed above, $n_{F}$ and $\gamma_{r}$ must be continuous functions of $\gamma$. A similar reasoning allows one to conclude that they must also be continuous functions of $h$. The continuity of $\gamma_{r}$ and $n_{F}$ is a key property, and will be used later in Section V to select the physically correct solution of (7) whenever indeterminacies arise. Secondly, in Section VI it will be argued that $n_{F}$ can be identified with the logarithm of the partition function for the RP problem, and the consequences of such identification will be discussed. Although this identification is not necessary for solving the RP problem, it provides interesting additional insight into this problem insofar it helps making a link with thermodynamics.

\section{B. Calculation of $\gamma_{r}$}

We now show how the density $\gamma_{r}(\gamma, h)$ of redundant bonds is calculated. Obviously when $\gamma=0$ there are no redundant bonds, i.e. $\gamma_{r}(\gamma=0, h)=0$, so we can write

$$
\gamma_{r}(\gamma, h)=\left.\int_{0}^{\gamma} \frac{\partial \gamma_{r}}{\partial \gamma}\right|_{h} d \gamma
$$

where the integral is done along a path of constant $h$. In Appendix $\mathrm{A}$ we show 66] that

$$
\left.\gamma \frac{\partial\left\langle B_{r}\right\rangle}{\partial \gamma}\right|_{h}=\left\langle B_{o v}\right\rangle,
$$

where $\left\langle B_{o v}\right\rangle_{\gamma}$ is the total number of overconstrained bonds in $\mathcal{B}$. Consider two randomly chosen sites $i$ and 
$j$. As discussed in Section IV a bond $b_{i j}$ is overconstrained if $i$ and $j$ are rigidly connected to the background, even in the absence of this bond. On a random graph, this happens with probability $R^{2}$. Thus a bond is present and overconstrained with probability $(\gamma / N) R^{2}$, and therefore the average number of overconstrained bonds is $\left\langle B_{o v}\right\rangle=\gamma R^{2} N / 2$. We can now write Eq. (24) as

$$
\left.\frac{\partial \gamma_{r}}{\partial \gamma}\right|_{h}=R^{2}
$$

so that now Eq. 23) reads

$$
\gamma_{r}(\gamma, h)=\int_{0}^{\gamma} R^{2}(\gamma, h) d \gamma
$$

Notice that, while (23) and (24) are exact for arbitrary graphs, 25) only holds under the assumption that two neighboring sites $i$ and $j$ are independently rigid with probability $R$. Thus in deriving (26), correlations have been ignored. This is correct on random graphs of the type considered here, but fails on finite dimensional lattices.

It is not immediately obvious how (26) can be integrated since we do not have an explicit expression for $R$ as a function of $\gamma$. However using $x=\gamma R=y-h$ one can write $R^{2} d \gamma=R d x-x d R=(R-(y-h) \partial R / \partial y) d y$, where $R=G_{g}(y)$ as given by (7). After integrating by parts,

$$
\gamma_{r}=\left.\left\{2 \int G_{g}(y) d y-(y-h) G_{g}(y)\right\}\right|_{y=h} ^{y=x+h}
$$

Using (3), Eq. (27) can be integrated to give

$$
\begin{aligned}
\gamma_{r} & =\left.\left\{(y+h-2 g) G_{g}(y)+2 g P_{g}(y)\right\}\right|_{y=h} ^{y=x+h} \\
& =(y+h) G_{g}(y)-2 h G_{g}(h)+2 g\left[G_{g+1}(h)-G_{g+1}(y)\right]
\end{aligned}
$$

This expression enables us to calculate the density of redundant bonds analytically. In previous work on Bethe lattices [20], (26) was solved in zero field by numerical integration, using for this purpose the values of $R$ that result from iteration of (2). Although the algebra becomes slightly more complicate, the approach used here to calculate $\gamma_{r}$ is applicable to Bethe lattices as well. Later in Section $\mathrm{\nabla B}$ we present some results for Bethe lattices.

\section{The density of zero modes}

Using (7), 21), (22), and (28) we can write

$$
n_{F}(\gamma, h)=\frac{\gamma}{2}\left(R^{2}-1\right)+(h-g)(R-1)+g P_{g}(y)
$$

where $y=x+h=\gamma R+h=\gamma(R+H)$. Because of (22), continuity of $\gamma_{r}$ implies that of $n_{F}$.

\section{A consistency check}

In the limit $\gamma \rightarrow N$, all bonds are present and there are no remaining degrees of freedom, i.e. $n_{F} \rightarrow 0$. It is easy to verify that Eq. (29) indeed satisfies this condition.

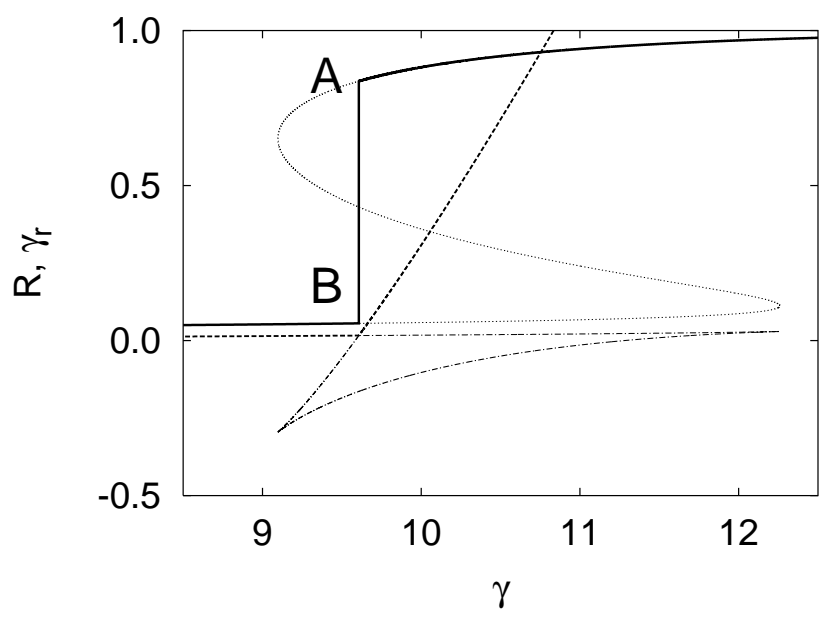

FIG. 4: The density of redundant bonds $\gamma_{r}$ (thick dashed) describes a Maxwell loop (dot-dashed) whose crossing point defines the location of the true transition. After discarding the unphysical loop, the density $R$ of rigid sites (thick solid) jumps at the transition between $\mathrm{A}$ and $\mathrm{B}$, but $\gamma_{r}$ is continuous. Shown is an example with $g=10$ and $h=5$.

\section{LOCATION OF THE TRANSITION POINT}

\section{A. The general case}

The value $\gamma_{c}(h)$ where the transition takes place is univocally determined by the requirement that $\gamma_{r}$ be a continuous function of $\gamma$. In order to see how this works, imagine that for a given $h<h *$ one lets $h<y<\infty$ and calculates $\gamma_{r}(y, h)$ (Eq. (28)) and $\gamma(y, h)$ (Eq. (10)), thus obtaining a plot of $\gamma_{r}$ vs $\gamma$ (Fig. (4). In doing so one finds that $\gamma_{r}$ describes a loop (sometimes called a "Maxwell loop" in Statistical Mechanics).

The existence of a crossing point implies that two values $y_{A}$ and $y_{B}$ exist such that

$$
\left.\left\{(y+h-2 g) G_{g}(y)+2 g P_{g}(y)\right\}\right|_{y=y_{A}} ^{y=y_{B}}=0
$$

where $y_{A, B}=\gamma_{c}(h) R_{A, B}+h$. Eq. (30) cannot be solved analytically in general, however it is easily solved numerically (by iteration) for each value of $h$ and $g$, to obtain $\gamma_{c}(g, h)$. In this fashion the location of the coexistence line can be calculated to arbitrary precision (Fig. 5). At the transition point $\gamma_{c}(h)$ the order parameter $R$ jumps (Fig. [6), but the density of redundant bonds $\gamma_{r}$ is continuous (Fig. 6b). 


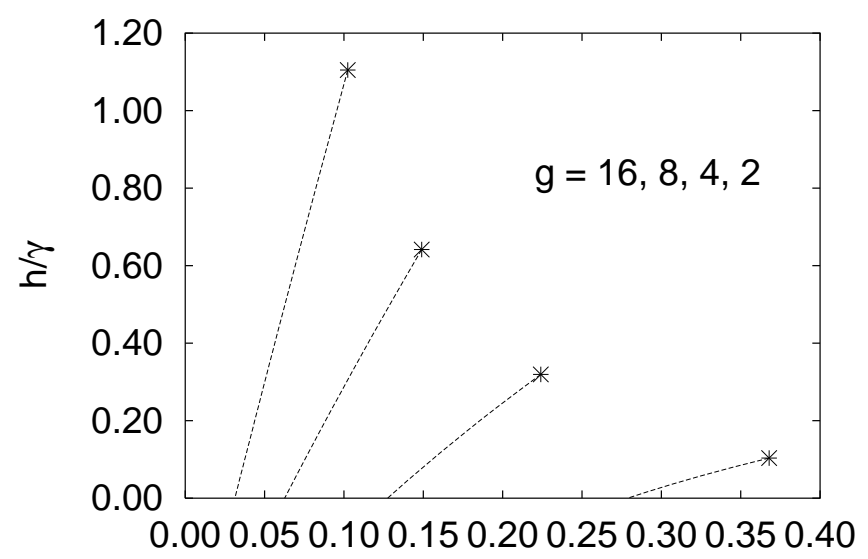

$1 / \gamma$

FIG. 5: Coexistence lines (lines of first-order phase transitions-dashed) in the space defined by the two intensive parameters of Rigidity Percolation: the "field" $H=h / \gamma$ and the "temperature" $1 / \gamma$, for several values of the number $g$ of degrees of freedom per site. The critical point, defined by (13), is indicated with an asterisk. For all $g \geq 2$, i.e. for Rigidity Percolation, this critical point has classical indices $\alpha=0, \beta=1 / 2, \gamma=1$ and $\delta=3$. The case $g=1$ (Scalar Percolation, not shown) has its critical point at $\gamma=1$ and $H=0$, and belongs to a different universality class, with $\alpha=-1, \beta=1, \gamma=1$ and $\delta=2$.

\section{B. The case of zero field. Maxwell counting on the $(g+1)$-rigid-core}

For zero field one has that $R=0$ for $\gamma<\gamma_{c}$. Eq. 26] then implies that $\gamma_{r}=0$ for $\gamma \leq \gamma_{c}$. At the transition point $\gamma_{c}$, a rigid cluster suddenly appears that contains a fraction $R_{c}$ of the graph. In zero field, Eq. (30) reads

$$
\left(x_{c}-2 g\right) R_{c}+2 g P_{g}\left(x_{c}\right)=0,
$$

where all quantities are evaluated in the rigid phase $(R$ and $y$ are zero in the floppy phase).

Even this simpler equation does not admit analytic treatment. However, very precise solutions can be obtained numerically as described already. Table \ contains some examples.

Please notice that $\gamma_{c}$ is smaller than the value $2 g$ needed for global balance of constraints and degrees of freedom. However, all degrees of freedom belonging to sites on the rigid cluster (which appears suddenly at the transition point) are by definition canceled out by constraints. Moreover, since for zero field the number of redundant constraints per site is exactly zero at the transition (See Fig. (6), one concludes that the average number of bonds per site, for sites on the rigid cluster, must be exactly $2 g$ at $\gamma_{c}$. The conclusion is then that sites on the rigid cluster have more bonds than average.

This observation may be formalized in the following way. Using $\gamma R=x, R=\sum_{k=g}^{\infty} P_{k}(x)$ with $P_{k}(x)=e^{-x} x^{k} / k !$, and $x P_{k}(x)=(k+1) P_{k+1}(x)$, condition (31) can be writ-
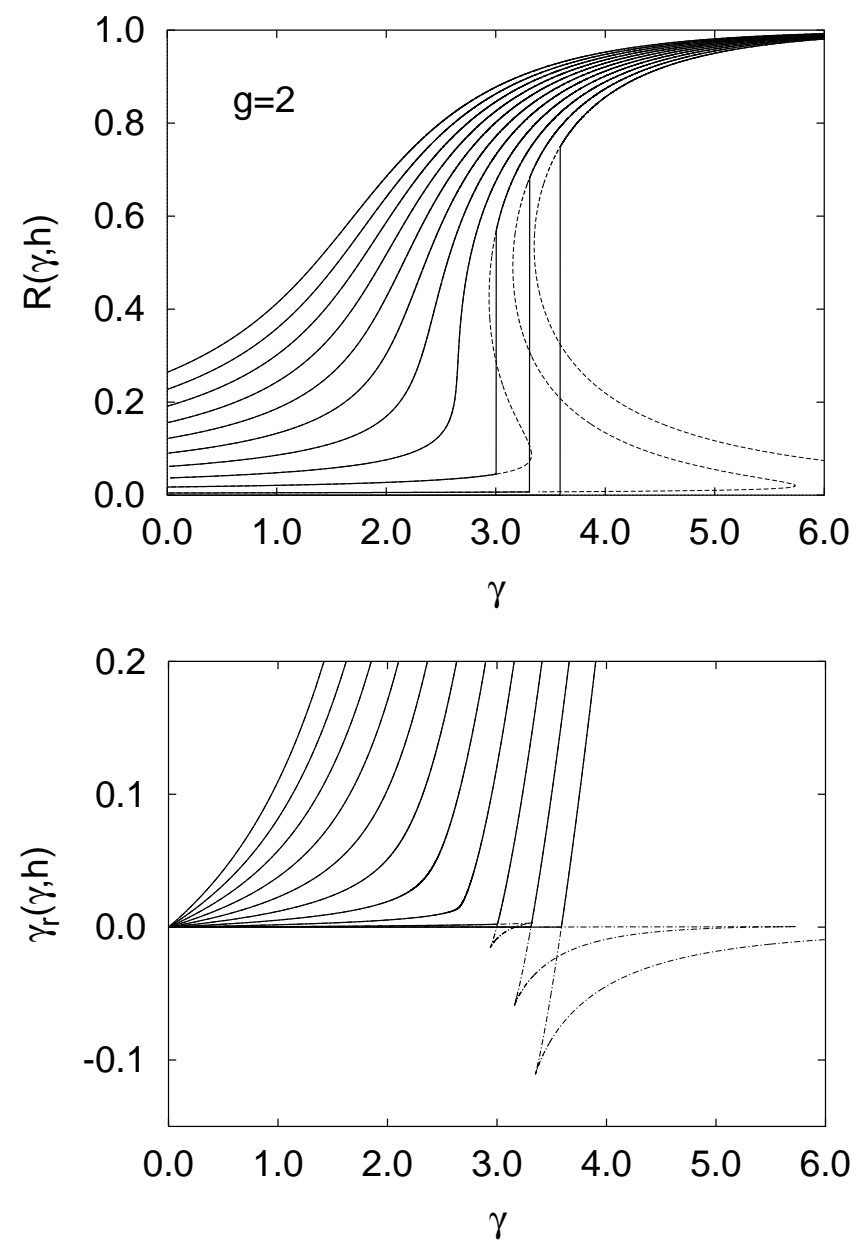

FIG. 6: a) The final values for the density of rigid sites $R$ (thick lines). The transition point $\gamma_{c}(h)$, where $R$ undergoes a discontinuous jump, is determined by the physical requirement that the density of redundant bonds $\gamma_{r}(\mathbf{b})$ ) be continuous. In this example $g=2$ and (from right to left): $h=0.0,0.1,0.20, \ldots, 1.0$. For $g=2$ the critical field is $h_{c}=0.287$.

ten

$$
\sum_{k=g+1}^{\infty}(2 g-k) P_{k}(x)=0,
$$

which in turn implies

$$
\frac{\sum_{k=g+1}^{\infty} k P_{k}(x)}{\sum_{k=g+1}^{\infty} P_{k}(x)}=2 g .
$$

This sum counts the number of rigid neighbors for sites on the $(g+1)$-rigid-core, that is, the subgraph of the rigid cluster that has minimum coordination $(g+1)$. Eq. (33) means that the $(g+1)$-rigid-core has an exact balance of degrees of freedom.

Clearly, the rigid cluster may also contain sites not in the $(g+1)$-rigid-core. When these are considered, it is possible to see that the balance of constraints and degrees of freedom is still respected. 


\begin{tabular}{ccc}
\hline \hline$g$ & $\gamma_{c}(g)$ & discontinuity $R_{c}$ \\
\hline 2 & $3.58804747296539 \ldots$ & $0.74915378510250 \ldots$ \\
\hline 3 & $5.7549256115462 \ldots$ & $0.8812398370507 \ldots$ \\
\hline 4 & $7.84295819428849 \ldots$ & $0.933538491167168 \ldots$ \\
\hline 5 & $9.89551361859910 \ldots$ & $0.959638549916489 \ldots$ \\
\hline 6 & $11.9288724790262765 \ldots$ & $0.974275720961391 \ldots$ \\
\hline$\rightarrow \infty$ & $2 g$ & 1.0 \\
\hline \hline
\end{tabular}

TABLE I: The Rigidity Percolation threshold coordination $\gamma_{c}$, and the jump in the rigid cluster density $R_{c}$ on Erdős-Renyi random graphs, obtained from solving (31) for several values of $g$, the number of degrees of freedom per site.

\section{The case of large $g$}

An analytic solution of Eq. (31) is possible (though somewhat trivial) in the $g>>1$ limit. In this case $P_{g} \rightarrow$ 0 and $R_{c} \rightarrow 1$, i.e. there is a jump from $R=0$ to $R=1$ at the transition point. Plugging these observations into (31) one gets, for zero field,

$$
\gamma_{c}=2 g \quad g>>1 .
$$

This means that the rigid transition happens exactly at the point where global balance between constraints and degrees of freedom is attained. In other words, at $\gamma_{c}$ Maxwell counting is exact on the rigid cluster for all $g$, while for $g>>1$ it is globally exact as well.

\section{Bethe lattices}

A graph where each site has exactly $z$ randomly chosen neighbors is topologically equivalent to a Bethe lattice, except for finite-size corrections. When this system is randomly diluted, one obtains what is called "random bond model" in Ref. [20], in which each of the $z$ bonds on a site is present with probability $p$. We will only state the final results for this system. In zero field the transition point $p_{c}$ is determined by

$$
\sum_{j=g+1}^{z}(j-2 g) Q_{j}^{(z)}\left(x_{c}\right)=0
$$

where $x=p T, Q_{j}^{(z)}(x)=\left(\begin{array}{c}z \\ j\end{array}\right) x^{j}(1-x)^{z-j}$ and $T$ satisfies the recursive equation [19]

$$
T=\sum_{j=g}^{z-1} P_{j}^{(z-1)}(x) .
$$

The transition condition (35) gives rise to a polynomial equation which is easily solved for small values of $g$ and $z$. For $g=2$ and $z=5$ the corresponding quadratic equation results in $p_{c}=0.83484234$. For $g=2$ and $z=6$ a cubic equation is obtained, and its solution is $p_{c}=0.656511134$. This last value is consistent with but more precise than $p_{c}=0.656$ as obtained in previous work 19] using numerically exact matching algorithms for RP [47, 50].

\section{RELATION BETWEEN $n_{F}$ AND A FREE ENERGY}

In this Section, the consequences of making the identification $n_{F} \rightarrow(\log \mathcal{Z}) / N$ are explored. It has been shown some time ago that SP can be mapped onto the $q \rightarrow 1$ limit of the Potts model[43, 44]. This mapping allows one to draw a parallel between an equilibrium thermodynamic transition (Potts) and percolation, which may be described as a purely geometric transition. One of the outcomes of this equivalence is the identification of the total number of clusters in percolation as the logarithm of the partition function for the resulting Potts model. The existence of this mapping has had profound impact on our understanding of scalar percolation, e.g. by allowing the exact calculation of percolation critical indices in two dimensions [2]. No equivalent mapping has been found for RP yet. However it has been suggested [20, 45] that a possible generalization for the free energy in RP for zero field is the total number of remaining degrees of freedom (zero modes or floppy modes). In the $g=1$ case of RP each cluster has one remaining degree of freedom, thus the SP result is recovered.

In Section IV it was shown that $n_{F}$ (equivalently $\gamma_{r}$ ) has to be continuous. This was used in Section $\nabla$ to locate the point $\gamma_{c}$ where the first-order transition happens. Thus $n_{F}$ plays a role similar to that of the Gibbs free energy, which is also a continuous function of its intensive parameters. In this section we add further evidence supporting the identification of $n_{F}$ as the logarithm of the "partition function", or free energy, for the RP problem on random graphs. We demonstrate that the ansatz $\mathcal{Z}_{R P} \propto e^{N n_{F}}$ is consistent also in the presence of a field, and show how several thermodynamic quantities result from derivatives of this free energy.

In Appendix A1 it is shown that the derivatives of $n_{F}$ are

$$
\begin{aligned}
& \left.\frac{\partial n_{F}}{\partial \gamma}\right|_{h}=\frac{1}{2}\left(R^{2}-1\right) \\
& \left.\frac{\partial n_{F}}{\partial h}\right|_{\gamma}=R-1,
\end{aligned}
$$

As expected, the order parameter $R$ results form deriving the logarithm of the partition function with respect to the field-like parameter, up to a constant shift.

From Eqns. (37) we furthermore obtain

$$
\begin{aligned}
\left.\frac{\partial n_{F}}{\partial \gamma}\right|_{H} & =\frac{1}{2}\left(R^{2}-1\right)+H(R-1) \\
\left.\frac{\partial n_{F}}{\partial H}\right|_{\gamma} & =\gamma(R-1)
\end{aligned}
$$




\section{A. Maxwell's Rule of Equal Areas}

The continuity requirement 30 employed in this work to locate the true transition point $\gamma_{c}$ is analogous to the condition that the free energy be continuous, as noted in previous work 20]. The continuity of an appropriate thermodynamic potential is discussed in elementary statistical mechanics textbooks in connection, for example, with Van der Waals' equation [46, 51], where it is used as a criterion to locate the transition. The continuity condition for our RP problem is written in general as

$$
\int_{A}^{B} d n_{F}=0
$$

where $A$ and $B$ are the two phases that coexist at the first-order transition (see Fig. 4). Clearly (30) is the particular case of (39) for which the integration is done along a path of constant field. Choosing a path $A \rightarrow B$ on which $\gamma$ is constant instead, it is easy to put (39) in the form of the famous Maxwell's "rule of equal areas". Start from

$$
0=\left.\int_{A}^{B} \frac{\partial n_{F}}{\partial h}\right|_{\gamma} d h
$$

where the integral is done along an "isotherm" (a line of constant $\gamma$ ). After using (37b) and integrating by parts, this reads

$$
h\left(R_{B}-R_{A}\right)=\int_{A}^{B} h(R, \gamma) d R
$$

where $h(R, \gamma)=G_{g}^{-1}(R)-\gamma R$.

Eq. (41) is equivalent to the condition $P\left(V_{B}-V_{A}\right)=$ $\int_{A}^{B} P(V, T) d V$ for a fluid, i.e. Maxwell's rule. Fig. 7 serves to illustrate the fact that the areas above and below the horizontal coexistence line are equal, as (41) requires.

\section{B. Energy, Entropy, and Work}

In the following we will assume that $n_{F}$ (Eq. (29)) is the logarithm of the partition function, i.e. $n_{F}=-\gamma f$ with $f$ a free-energy density, and $\gamma=1 / T$ the inverse temperature. The energy density $e$ is then

$$
e=-\left.\frac{\partial n_{F}}{\partial \gamma}\right|_{H}=\frac{1}{2}\left(1-R^{2}\right)+H(1-R) .
$$

The entropy per site $s$ turns out to be

$$
s=-\left.\frac{\partial f}{\partial T}\right|_{H}=n_{F}+\gamma e=g\left[P_{g}(y)-(R-1)\right] .
$$

From the first law of thermodynamics, and using Eqns. (42) and (43), we conclude that, in an infinitesimal

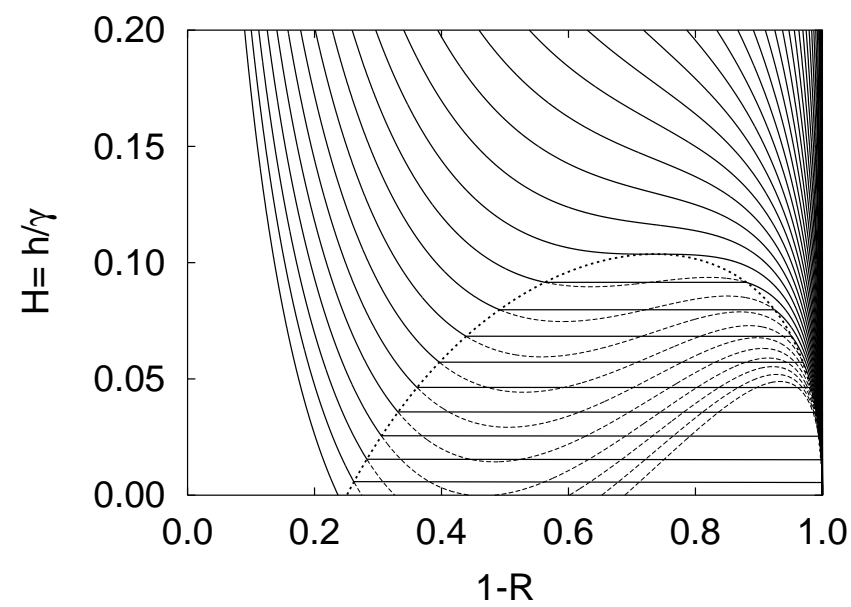

FIG. 7: This plot of the field $H$ versus the order parameter $R$ for Rigidity Percolation ( $g=2$ in this example) is the equivalent of a $P-V$ diagram for fluids. Shown are the "isotherms" (solid lines) along which the coordination number $\gamma$ is constant. The thick dashed line delimits the "coexistence" region, and was obtained by solving (41) numerically. The areas delimited by the thin dashed lines above and below the (solid) horizontal coexistence line are equal.

transformation, the "work" done by the system against the environment is:

$$
d w=d q-d e=\frac{1}{\gamma} d s-d e=\frac{1}{\gamma} \frac{\partial n_{F}}{\partial H} d H=(R-1) d H
$$

The analogous of the constant-pressure heat capacity for a fluid is in our case the constant-field heat capacity

$$
c_{H}=-\left.\gamma \frac{\partial s}{\partial \gamma}\right|_{H}=-\left.\gamma^{2} \frac{\partial e}{\partial \gamma}\right|_{H}=\frac{y^{2} P_{g-1}(y)}{1-\gamma P_{g-1}(y)},
$$

which is nonnegative by (18), and diverges as $\left|\gamma-\gamma^{*}\right|^{-1}$ at the critical point. This of course should not be taken to mean that $\alpha=1$, since it is the constant-density heat capacity what defines $\alpha$ (For a discussion see Ref. [52]). The constant-field heat capacity $c_{H}$ is expected to diverge with the same exponent $\gamma$ as the susceptibility, unless the system has certain symmetries, which is the case for the Ising model, but not for RP.

The analogous of the "constant-density" heat capacity is

$$
c_{R}=-\left.\gamma \frac{\partial s}{\partial \gamma}\right|_{R},
$$

which is zero and thus $\alpha=0$.

\section{Clausius-Clapeyron Equation}

Let $\Delta f=-1 / \gamma \Delta n_{F}=-1 / \gamma\left(n_{F}(B)-n_{F}(A)\right)$ be the free-energy difference between the two phases in the coexistence region. This is a function of the "temperature" 
$T=1 / \gamma$ and the field $H=h / \gamma$. Following standard texts [53], we write

$$
\left.\frac{\partial H}{\partial T}\right|_{\Delta f}=-\frac{\left.\frac{\partial \Delta f}{\partial T}\right|_{H}}{\left.\frac{\partial \Delta f}{\partial H}\right|_{T}}=-\frac{\Delta s}{\Delta R},
$$

where the derivative on the left-hand side is evaluated on any line of constant $\Delta f$. Using (43) one can write $\Delta s=g\left(\Delta P_{g}-\Delta R\right)$. On the coexistence line, $\Delta f=$ 0 so $\left.\Delta s\right|_{\text {coex }}=\left.\gamma \Delta e\right|_{\text {coex }}$ and, using (42) we have that $\left.\Delta s\right|_{\text {coex }}=-h \Delta R-\gamma / 2 \Delta\left(R^{2}\right)=-\Delta R\left(\frac{y_{A}+y_{B}}{2}\right)$. Thus the Clausius-Clapeyron equation for the RP problem can be written as

$$
\left.\frac{\partial H}{\partial T}\right|_{\text {coex }}=\frac{y_{A}+y_{B}}{2}
$$

Since on approach to the critical point, $y \rightarrow y^{*}=g-1$ (Eq. (13)), we conclude that the coexistence line reaches the critical point with slope $(g-1)$ in the $H-T$ plane

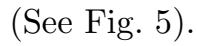

\section{DISCUSSION}

The Rigidity Percolation problem with $g$ degrees of freedom per site has been considered on Erdös-Renyi graphs with average coordination $\gamma$. An external field $h$ is introduced by connecting each site to a rigid background, or "ghost site", with a certain probability that depends on $h$ as described in Section IIC The resulting Equation of State (7) for the density $R$ of sites that are rigidly connected to the background, undergoes a firstorder phase transition on a "coexistence line" $\gamma_{c}(h)$. This line ends at a critical point (Fig. 5) with classical exponents $\alpha=0, \beta=1 / 2, \gamma=1$ and $\delta=3$. For comparison, the critical point of Scalar percolation (the case of $g=1$ ) is located at $h=0$ and has different critical exponents: $\alpha=-1, \beta=1, \gamma=1$ and $\delta=2[1]$. Therefore in the MF approximation, Scalar and Rigidity percolation are in different universality classes. In two dimensions, a similar conclusion is reached by numerical means [15, 45], were both transitions are continuous in zero field.

It has been recently argued 54 that for certain spin systems, the existence of a discontinuous transition in the MF approximation is enough to ensure that, in finite space dimensions $d$, there is a discontinuous transition if $d$ is large enough. If a this result holds for RP, one would expect to see a discontinuous transition in zero field for $d$ (and perhaps $g$ ) large enough. Up to now, extensive numerical simulations for RP have been performed only in two dimensions 15, 45, 48, 55. Except for pathological cases where the transition happens at zero dilution [15], it seems that the transition is continuous for all $g$ in two dimensions. Large scale simulations are still needed to clarify this issue.

On Cayley Trees with a boundary, $g$-rigidity percolation(RP) is closely related to $(g+1)$-bootstrap percolation(BP) 19, 34]. Both percolate at the same density $p$ of present bonds, although with different spanning cluster densities on trees. On random graphs, which have the local structure of a tree but no boundaries, $k$-core transition stays unchanged, but the RP transition is delayed to larger $p$ values [19]. We have explicitely shown that, at the critical concentration $\gamma_{c}$ where the rigid cluster first appears, it has an exact balance of degrees of freedom, i.e. it has exactly $g$ bonds per site. This condition is satisfied both on the whole rigid cluster and on its $(g+1)$-rigid core. However globally the system has lesser bonds than needed to attain this balance, meaning that the rigid cluster is selectively made out of sites with more bonds than average.

In previous work on Bethe lattices in zero field [20] it was suggested that the number $n_{F}$ of uncanceled degrees of freedom is a free energy for the RP problem. For SP $(g=1)$ this result holds exactly, being one of the outcomes of the Fortuyn-Kasteleyn Random-Cluster model [3, 44], which contains SP and the Potts model as particular cases. For RP, however, this identification only has the status of a plausible ansatz. This ansatz was used recently to predict some thermodynamic properties of chalcogenide glasses [56], for which the RP transition has been shown to be relevant [11, 14, 57, 58].

Some of the reasons to believe that this identification might be correct in general are: a) the fact that $n_{F}$ must be continuous at the discontinuous RP transition, and, b) the fact that for $g=1, n_{F}$ is the number of connected clusters per site, so the FK result for SP is recovered exactly. In this work we have shown that, in the presence of a properly defined field $h$, the ordered parameter $R$ can be obtained as a derivative of the free energy with respect to $h$, thus adding further support to the belief that this identification is correct.

Under the assumption that $\log \mathcal{Z}=N n_{F}$, the entropy per site $s$ can be derived. The resulting expression (43) was found to depend on the order parameter $R$ alone. This is a property of other MF systems like for example the Ising ferromagnet.

On the pedagogical side, we have attempted to situate the discussion in terms of the parallel between the $\mathrm{RP}$ transition in a field and a condensation transition. Since only topological (connectivity) properties are important for RP, it can be said that, in a sense, RP in a field is a sort of "geometric condensation transition". Fig. 7 illustrates the similarities between both transitions. However a closer match is possible. Comparing (7) to (9) one notices that, in a MF condensation transition, $\lambda \propto \rho^{2}=1 / v^{2}$ plays the role of the order parameter $R$ in RP. From this point of view, the analog of a "volume" in RP would be $1 / R^{1 / 2}$. The analog of a $P-V$ plot is shown in Fig. 8 Notice however that, within this definition of a "volume", $P(V)$ does not satisfy Maxwell's rule of equal areas, $\int_{A}^{B} V(P) d P=0$. 


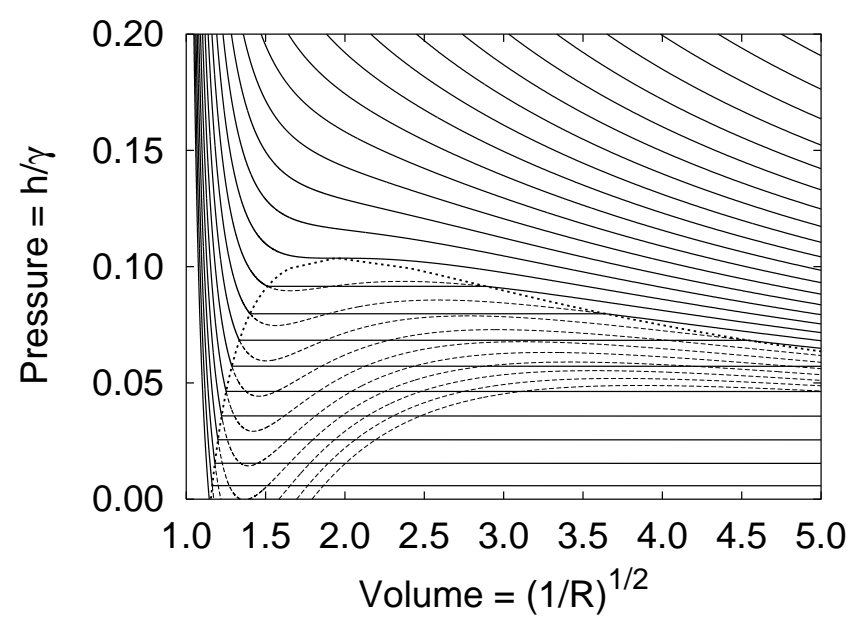

FIG. 8: Illustration of the similarities between RP and a condensation transition. In this plot, $H=h / \gamma$ is taken to be the analog of a pressure and $1 / R^{1 / 2}$ the analog of a volume. The data are the same as shown in Fig. 7

\section{Acknowledgments}

The author has benefited from many useful discussions with Prof. Phillip Duxbury in the early stages of this work. Financial support from the SNI program of CONACYT, and from a CONACYT grant for research project $36256-\mathrm{E}$, is acknowledged.

\section{APPENDIX A: DERIVATIVES OF THE NUMBER OF REDUNDANT BONDS}

Let $\mathcal{F}(\mathcal{B})$ be an arbitrary function of the set of present edges $\mathcal{B}$ of a graph $\mathcal{G}$, and let $P(\mathcal{B})$ be the probability to have edge set $\mathcal{B}$. The average of $\mathcal{F}$ is defined as

$$
\langle\mathcal{F}\rangle=\sum_{\{\mathcal{B}\}} P(\mathcal{B}) \mathcal{F}(\mathcal{B})
$$

where $\sum_{\{\mathcal{B}\}}$ is a sum over all configurations of present edges.

If each edge is independently present with probability $p$ and absent with probability $1-p$, then clearly

$$
P_{p}(\mathcal{B})=p^{|\mathcal{B}|}(1-p)^{\left|\mathcal{B}_{\max }\right|-|\mathcal{B}|}
$$

where $\mathcal{B}_{\text {max }}$ is the edge set of maximum possible cardinality.

We now write $p=p_{1} p_{2}$ with $0 \leq p_{1}, p_{2} \leq 1$. This can be realized by assuming that two types of bonds are independently present with probabilities $p_{1}$ and $p_{2}$ respectively, and that in order for an edge to be "active", both types of bonds must be present on that edge. $\mathcal{F}$ is now a function of active bonds only, which are present with probability $p_{1} p_{2}$, so that one can write

$$
\begin{aligned}
\langle\mathcal{F}\rangle_{p_{1} p_{2}} & =\sum_{\{\mathcal{B}\}} P_{p_{1}}(\mathcal{B}) \sum_{\left\{\mathcal{B}^{\prime}\right\} \subseteq \mathcal{B}} P_{p_{2}}\left(\mathcal{B}^{\prime}\right) \mathcal{F}\left(\mathcal{B}^{\prime}\right) \\
& =\sum_{\{\mathcal{B}\}} P_{p_{1}}(\mathcal{B}) S\left(p_{2}, \mathcal{B}\right)
\end{aligned}
$$

Notice that $P_{p_{2}}\left(\mathcal{B}^{\prime}\right)$ has a factor $p_{2}$ for each present bond in configuration $\mathcal{B}^{\prime}$, and a factor $1-p_{2}=q_{2}$ for each bond in $\mathcal{B}$ which is absent in $\mathcal{B}^{\prime}$. Factors associated with bonds not in $\mathcal{B}$ can be summed out to give one and therefore need not be considered. Thus if $|\mathcal{B}|$ is the number of present bonds in configuration $\mathcal{B}$ and $\left|\mathcal{B}^{\prime}\right|$ the number in $\mathcal{B}^{\prime}, P_{p_{2}}\left(\mathcal{B}^{\prime}\right)=p_{2}^{\left|\mathcal{B}^{\prime}\right|} q_{2}^{|\mathcal{B}|-\left|\mathcal{B}^{\prime}\right|}$.

Now let $p_{2} \rightarrow 1$. In this case we can expand $S\left(p_{2}, \mathcal{B}\right)$ in powers of $q_{2}$. The zeroth-order contribution comes from $\mathcal{B}^{\prime} \equiv \mathcal{B}$, the first-order contribution from the $|\mathcal{B}|$ configurations $\mathcal{B}^{\prime}$ which have exactly one bond $b$ less than $\mathcal{B}$, etc.

$$
\begin{aligned}
S\left(p_{2}, \mathcal{B}\right) & =p_{2}^{|\mathcal{B}|} \mathcal{F}(\mathcal{B})+q_{2} p_{2}^{|\mathcal{B}|-1} \sum_{b \in \mathcal{B}} \mathcal{F}(\mathcal{B}-b)+ \\
& +q_{2}^{2} p_{2}^{|\mathcal{B}|-2} \sum_{b, b^{\prime} \in \mathcal{B}} \mathcal{F}\left(\mathcal{B}-b-b^{\prime}\right)+\ldots,
\end{aligned}
$$

where we have written $\mathcal{B}-b$ to denote the result of deleting bond $b$ from $\mathcal{B}$. To first order in $q_{2}$,

$$
S\left(p_{2}, \mathcal{B}\right)=\mathcal{F}(\mathcal{B})+q_{2} \sum_{b \in \mathcal{B}}(\mathcal{F}(\mathcal{B}-b)-\mathcal{F}(\mathcal{B})),
$$

therefore

$$
\langle\mathcal{F}\rangle_{p_{1} p_{2}}=\langle\mathcal{F}\rangle_{p_{1}}+q_{2}\left\langle\sum_{b \in \mathcal{B}}(\mathcal{F}(\mathcal{B}-b)-\mathcal{F}(\mathcal{B}))\right\rangle_{p_{1}} .
$$

Taking derivatives with respect to $p_{2}$ and letting $p_{2}=1$ one finally finds that, for arbitrary $\mathcal{F}$,

$$
p \frac{\partial\langle\mathcal{F}\rangle}{\partial p}=\left\langle\sum_{b \in \mathcal{B}}\{\mathcal{F}(\mathcal{B})-\mathcal{F}(\mathcal{B}-b)\}\right\rangle .
$$

Eq. (A7) generalizes previous results of Coniglio in Ref. [59], and is the tool we use in this Section to derive some important relations.

Consider now the case in which $\mathcal{F}=B_{r}(\mathcal{B})$, the total number of redundant constraints. In order to calculate the derivative of $B_{r}$ with respect to $p$, we notice that when removing a bond $b$ from a configuration $\mathcal{B}$ of present bonds, the total number of redundant constraints will be reduced by one if and only if $b$ is overconstrained. Otherwise if $b$ is not overconstrained, $B_{r}$ remains unchanged by the removal of $b$. Eq. (A7) then implies that

$$
p \frac{\partial\left\langle B_{r}\right\rangle}{\partial p}=\gamma \frac{\partial\left\langle B_{r}\right\rangle}{\partial \gamma}=\left\langle B_{o v}\right\rangle,
$$

where $\left\langle B_{o v}\right\rangle$ is the average number of overconstrained bonds in $\mathcal{B}$. 
A calculation of the field derivative of $\left\langle B_{r}\right\rangle$ requires some additional considerations. Eq. (A7) was derived under the assumption that each edge is independently present with some fixed probability, while our definition of ghostfield in Section $\Pi \mathrm{C}$ implies that each site can have any number $n$ of ghost constraints with poissonian probability $P_{n}(h)=e^{-h} h^{n} / n$ !. We can represent this poissonian process in a way that enables us to use (A7) as follows: we assume that each site has $M>>h$ slots connecting it to the background, and that each slot is occupied by one ghost edge with probability $p_{h}=h / M$. For large $M$, the probability to have $n$ ghost edges on a site is $P_{n}(h)$, so one has a realization of the poissonian distribution. This equivalent system has a total of $N M$ ghost slots, each occupied by a ghost bond with a small probability $p_{h}$, so we can now use Eq. A77.

Eliminating a present ghost edge reduces the number of redundant constraints by one if both of the following conditions are satisfied: a) the remaining constraints that this site has are enough to rigidize (i.e. $k_{\gamma}+k_{h} \geq g$ ), and b) the remaining ghost-constraints that this site has are not enough to rigidize on their own (i.e. $k_{h}<g$ ). The joint probability for these two conditions to be satisfied is

$$
\begin{aligned}
& \sum_{j=0}^{g-1} P_{j}(h) \sum_{k=g-j}^{\infty} P_{k}(x)= \\
& \sum_{j=0}^{g-1} P_{j}(h)-\sum_{j=0}^{g-1} P_{j}(h) \sum_{k=0}^{g-j-1} P_{k}(x)= \\
& R(\gamma, h)-R(0, h)=R-R_{h},
\end{aligned}
$$

where we have defined $R_{h}=G_{g}(h)$ as the density of rigid sites in the presence of the field $h$, for a graph with no edges $(\gamma=0)$. The total number of ghost slots is $N M$ and thus the total number of present ghost bonds which upon removal produce a change in the number of redundant constraints is on average

$$
N M \times \frac{h}{M} \times\left(R-R_{h}\right)
$$

Eq. (A7), with $p_{h}=h / M$, now implies that

$$
\frac{\partial\left\langle B_{r}\right\rangle}{\partial h}=N\left(R-R_{h}\right) .
$$

Using $\left\langle B_{r}\right\rangle_{\gamma}=\gamma_{r} N / 2$ one then has

$$
\frac{\partial \gamma_{r}}{\partial h}=2\left(R-R_{h}\right)
$$

\section{Checking derivatives of $\gamma_{r}$}

It is a trivial exercise to show that $\gamma_{r}$, as given by (28) has the right derivatives, i.e. satisfies

$$
\frac{\partial \gamma_{r}}{\partial \gamma}=R^{2}
$$

in agreement with (25), and

$$
\frac{\partial \gamma_{r}}{\partial h}=2\left\{R-R_{h}\right\}
$$

in agreement with (A12). Now since $g_{\text {eff }}=g(1-$ $\left.G_{g+1}(h)\right)-h\left(1-G_{g}(h)\right)$ one has that $\partial g_{\text {eff }} / \partial h=G_{g}(h)-$ $1=R_{h}-1$. Therefore the derivatives of $n_{F}$ (Eq. (22) are

$$
\begin{aligned}
& \frac{\partial n_{F}}{\partial \gamma}=\frac{R^{2}-1}{2} \\
& \frac{\partial n_{F}}{\partial h}=R-1
\end{aligned}
$$

\section{APPENDIX B: DERIVATION OF CRITICAL INDICES}

Eqns. (12a) and (12b) imply that, for $y \approx y^{*}$,

$$
R=G_{g}(y) \approx R^{*}+\frac{\Delta y}{\gamma^{*}}+\mathcal{O}\left((\Delta y)^{3}\right),
$$

with $\Delta y=y-y^{*}$, and $y^{*}=\gamma^{*} R^{*}+h^{*}$. The coefficient of the $\mathcal{O}\left((\Delta y)^{3}\right)$ term is nonzero. Furthermore $\Delta y=$ $\Delta h+\gamma^{*} \Delta R+R \Delta \gamma$, and Eq. (B1) can be rewritten as

$$
\begin{array}{rlrl}
\gamma^{*} R & =\gamma^{*} R^{*}+x-x^{*}+\Delta h+\mathcal{O}\left((\Delta y)^{3}\right) & \Rightarrow \\
R \Delta \gamma+\Delta h & =\mathcal{O}\left((\Delta y)^{3}\right) .
\end{array}
$$

On the critical isotherm $(\Delta \gamma=0)$ we thus have that $\Delta h \sim\left(\gamma^{*} \Delta R+\delta h\right)^{3} \Rightarrow \Delta R \approx(\Delta h)^{1 / 3}$, so $\delta=3$.

When $h<h^{*}$ the order parameter develops a $\gamma$-driven discontinuity $\Delta R \propto(\Delta h)^{\beta}$, which can be estimated in the following way. Assume $R^{*}$ is one of the three solutions of Eq. (B1) when $\Delta h<0$, and determine the value of $\gamma$ for which this happens. Because $\Delta y=0$ and $\Delta R=0$, one finds that $\Delta \gamma=-1 / R^{*} \Delta h$. Plugging this result back into Eq. (B1) and eliminating the solution $R=R^{*}$ one finds that the other two solutions behave as $\Delta R \propto(\Delta h)^{1 / 2}$, or $\beta=1 / 2$.

The "susceptibility" $\chi=\left.\frac{\partial R}{\partial h}\right|_{h^{*}}$ diverges on approach to the critical point as $\chi \propto(\Delta \gamma)^{-\gamma}$. Deriving Eq. (7) one gets

$$
\frac{\partial R}{\partial h}=\frac{P_{g-1}}{1-\gamma P_{g-1}}
$$

and recalling that $\gamma^{*}=\left(P_{g-1}\left(y^{*}\right)\right)^{-1}$ we find that $\chi \propto$ $(\Delta \gamma)^{-1}$, or $\gamma=1$.

Notice that Rushbrooke's $(\alpha+2 \beta+\gamma=2)$ and Griffith's $(\alpha+\beta(\delta+1))=2)$ relations are satisfied with $\alpha=0$, and this is consistent with the fact that the constant- $R$ specific heat (46) is zero. 
[1] D. Stauffer and A. Aharony, Introduction to Percolation Theory (Taylor and Francis, Bristol, 1994), 2nd. ed.

[2] A. Bunde and S. Havlin, eds., Fractals and Disordered Systems (Springer Verlag, Heidelberg, 1996), second ed.

[3] A. CONIGLIO and W. KLEIN, J. Phys. A-Math. Gen. 13(8), 2775 (1980).

[4] W. KLEIN and A. CONIGLIO, Phys. Lett. A 84(2), 83 (1981).

[5] A. CONIGLIO and F. PERUGGI, J. Phys. A-Math. Gen. 15(6), 1873 (1982).

[6] M. Sahimi, Applications of Percolation Theory (Taylor and Francis, Bristol, 1994), first ed.

[7] Sahimi, M, Phys. Rep.-Rev. Sec. Phys. Lett. 306(4-6), 214 (1998).

[8] S. FENG and P. SEN, Phys. Rev. Lett. 52(3), 216 (1984).

[9] Y. KANTOR and I. WEBMAN, Phys. Rev. Lett. 52(21), 1891 (1984).

[10] S. FENG, M. THORPE, and E. GARBOCZI, Phys. Rev. B-Condens Matter 31(1), 276 (1985).

[11] THORPE, MF, J. Non-Cryst. Solids 76(1), 109 (1985).

[12] A. DAY, R. TREMBLAY, and A. TREMBLAY, Phys. Rev. Lett. 56(23), 2501 (1986).

[13] M. THORPE and E. GARBOCZI, Phys. Rev. B-Condens Matter 35(16), 8579 (1987).

[14] M. Thorpe and P. M. Duxbury, eds., Rigidity Theory and Applications, Fundamental Materials Research (Kluwer Academic \& Plenum Press, New York, 1999).

[15] C. Moukarzel and P. Duxbury, Phys. Rev. E 59(3), 2614 (1999).

[16] CRAPO, H, Struct. Topol. 1(1), 26 (1979).

[17] Whiteley, W, Trans. Am. Math. Soc. 285(2), 431 (1984).

[18] HENDRICKSON, B, SIAM J. Comput. 21(1), 65 (1992).

[19] C. Moukarzel, P. Duxbury, and P. Leath, Phys. Rev. E 55(5), 5800 (1997).

[20] P. Duxbury, D. Jacobs, M. Thorpe, and C. Moukarzel, Phys. Rev. E 59(2), 2084 (1999).

[21] WU, FY, Rev. Mod. Phys. 54(1), 235 (1982).

[22] B. Bollobas, G. Grimmett, and S. Janson, Probab. Theory Relat. Field 104(3), 283 (1996).

[23] F. Karsch, C. Schmidt, and S. Stickan, Comput. Phys. Commun. 147(1-2), 451 (2002).

[24] Satz, H, Fortschritte Phys.-Prog. Phys. 50(5-7), 541 (2002).

[25] F. Karsch and S. Stickan, Phys. Lett. B 488(3-4), 319 (2000).

[26] S. Fortunato and H. Satz, Nucl. Phys. B-Proc. Suppl. 106, 890 (2002).

[27] KIRKPATRICK, S, Phys. Rev. Lett. 36(2), 69 (1976).

[28] M. Giri, M. Stephen, and G. Grest, Phys. Rev. B 16(11), 4971 (1977).

[29] Stephen, MJ, Phys. Rev. B 15(12), 5674 (1977).

[30] P. Reynolds, H. Stanley, and W. Klein, J. Phys. A-Math. Gen. 11(8), L199 (1978).

[31] H. Nakanishi and P. Reynolds, Phys. Lett. A 71(2,3), 252 (1979).

[32] ESSAM, JW, Rep. Prog. Phys. 43(7), 833 (1980).

[33] P. Reynolds, H. Stanley, and W. Klein, Phys. Rev. B 21(3), 1223 (1980).

[34] J. Chalupa, P. L. Leath, and G. R. Reich, J. Phys. C 12, L31 (1979).
[35] ADLER, J, Physica A 171(3), 453 (1991).

[36] M. KAUFMAN and D. STAUFFER, J. Stat. Phys. 73(56), 843 (1993).

[37] M. de Menezes and C. Moukarzel, Phys. Rev. E 60(5), 5699 (1999).

[38] B. Bollobás, in Graph Theory and Combinatorics, edited by B. Bollobás (Academic Press, 1984), pp. 35-57, proc. Cambridge Combinatorial Conf. in honour of Paul Erdös.

[39] LUCZAK, T, Discret. Math. 91(1), 61 (1991).

[40] Molloy, M, Random Struct. Algorithms 8(2), 159 (1996).

[41] B. Pittel, J. Spencer, and N. Wormald, J. Comb. Theory Ser. B 67(1), 111 (1996).

[42] B. Bollobás, Random Graphs (Cambridge University Press, Cambridge, 2001), 2nd ed.

[43] P. KASteleyN and C. FORTUIN, J. Phys. Soc. Jpn. S 26, 11 (1969).

[44] C. FORTUIN and P. KASTELEYN, PHYSICA 57(4), 536 (1972).

[45] D. Jacobs and M. Thorpe, Phys. Rev. E 53(4), 3682 (1996).

[46] H. E. Stanley, Introduction to Phase Transitions and Critical Phenomena, Internat.ional Series of Monographs on Physics (Oxford University Press, 1987).

[47] Moukarzel, C, J. Phys. A-Math. Gen. 29(24), 8079 (1996).

[48] D. JACOBS and M. THORPE, Phys. Rev. Lett. 75(22), 4051 (1995).

[49] S. Cannas, A. de Magalhaes, and F. Tamarit, Phys. Rev. B 61(17), 11521 (2000).

[50] D. Jacobs and B. Hendrickson, J. Comput. Phys. 137(2), 346 (1997).

[51] J. J. Binney, N. J. Dowrick, A. J. Fisher, and M. E. J. Newman, The Theory of Critical Phenomena: An introduction to the Renormalization Group, Oxford Science Publications (Oxford University Press, New York, 2002).

[52] D. A. Lavis and G. M. Bell, Statistical Mechanics of Lattice Systems, Texts and Monographs in Physics (Springer-Verlag, Berlin, 1999).

[53] K. Huang, Statistical Mechanics (John Wiley \& Sons, New York, 1987), 2nd ed.

[54] M. Biskup and L. Chayes, Rigorous analysis of discontinuous phase transitions via mean-field bounds (2002), commun. Math. Phys, to appear. DOI 10.1007/s00220003-0828-2, arXiv:math.PR/0207242.

[55] C. MOUKARZEL and P. DUXBURY, Phys. Rev. Lett. 75(22), 4055 (1995).

[56] Naumis, GG, Phys. Rev. B 61(14), R9205 (2000).

[57] PHILliPS, JC, J. Non-Cryst. Solids 34(2), 153 (1979).

[58] J. PHILLIPS and M. THORPE, Solid State Commun. 53(8), 699 (1985).

[59] CONIGLIO, A, J. Phys. A-Math. Gen. 15(12), 3829 (1982).

[60] See Refs. 8, 9, 15, 45], and also C. Moukarzel and P. Duxbury in [14].

[61] However, the rigid cluster is not a subset of the $(g+1)$-core

[62] For a discussion see for example [4].

[63] C. Moukarzel, to be published.

[64] This rigid background replaces the "ghost-site" in SP. For an alternative way to introduce a field, see [27]

[65] Or zero modes, or floppy modes. 
[66] For alternative derivations see [20, 45] 